\title{
Overview of Electron Sources and Stability Improvements on Vogel Mounted Thermal Sources
}

\author{
W.A. Mackie, G.G. Magera, and C.L. Fast
}

Applied Physics Technologies, Inc., 1600 NE Miller Street, McMinnville, OR 97128

We produce single crystal tungsten, transition metal carbides, and rare-earth hexaboride materials for electron sources. The rare-earth hexaborides[1], $\mathrm{LaB}_{6}$ and $\mathrm{CeB}_{6}$, are used commercially in a wide array of SEM, TEM, and lithography systems. Our research covers emission from singlecrystal boride and carbide thermionic and field emitters[2,3]. These materials have electron emission properties making them attractive candidates for stable emission sources in moderate vacuum applications. $\mathrm{CeB}_{6}(310)$ has a work function of $2.6 \mathrm{eV}$ and a relatively low evaporation rate in low $10^{-7}$ Torr pressures and at $<1800 \mathrm{~K}$ giving commercial lifetimes of $>2000$ hours. However, there are stability and brightness limitations needed for certain applications; new lithography and other uses have increased the need for emission stability beyond what is required for imaging applications.

We first describe a simple thermionic electron source that has several unique properties, including a defined emission area without edge effects and that when mounted in certain configurations has a planar electric field across the emitting surface. These features are beneficial for a range of applications including $\mathrm{x}$-ray generation, electron microscopy, lithography, and microwave devices. The source is a $\mathrm{CeB}_{6}(310)$ cylinder mounted flush in a specially shaped carbon guard-ring[4]. We make these carbon guard-ring structures from graphite, then mounted in a conventional mini-Vogel system, using pyrolytic carbon blocks as heating element (see Fig. 1). The work function of the carbon guard ring is $>1.5 \mathrm{eV}$ higher thereby suppressing emission from its surface and limiting emission to the $\mathrm{CeB}_{6}$ surface. Cathodes have been built with source diameters from $50 \mu \mathrm{m}$ to $1 \mathrm{~mm}$.

Secondly, we report on efforts to understand and enhance the emission stability of Vogel mounted sources. This has entailed understanding the interactions of boron from the metal boride with the carbon in the graphite blocks as well as the carbon-metal interactions between the heating blocks and the support post material. Barriers have been added to the Vogel mounted structure which has increased stability by evidently reducing chemical interactions found at high operating temperatures.

Figure 2 illustrates two changes to the mini Vogel mounted (MVM) thermionic cathode allowing better temperature stability and hence greater emission stability. First, by removing the $\mathrm{LaB}_{6}$ or $\mathrm{CeB}_{6}$ from between the pyrolytic graphite (PG) heater blocks and embedding or mounting the crystal in the top of a graphite blank, the interaction between the PG and the active metal elements of the crystal are eliminated from the heater circuit. In a standard MVM cathode, interaction of the crystal and PG results in resistance change over time which leads to a temperature change of the cathode. Figure 3 shows the change in resistance for $\mathrm{LaB}_{6}$ cathode and graphite blank cathode. The graph shows that with the graphite blank stability is much better when compared to the standard cathode. During operation the temperature at the tip of the Mo/Re clamping post can reach over $1000{ }^{\circ} \mathrm{C}$. It is believed that the high temperature leads to carbonization of post materials along the post-PG interface. In turn this process causes fluctuations in the resistance of the heater circuit. 
Placing a Re shim or barrier between the PG block and the post eliminates the resistance fluctuations for a graphite-metal interface operating at high temperatures.

[1] W.A. Swanson, et al., Surf. Sci., 83 11-28 (1979).

[2] W.A. Mackie, et al., J. Vac. Sci. \& Tech. B, 12 (2) Mar/Apr (1994).

[3] R.J. Kagarice, et al., J. Vac. Sci. \& Tech. B, 26 (2), Mar/Apr (2008).

[4] W.A. Mackie, et al., IVEC/IVESC '06 Conference Proceeding, IEEE Elect. Dev. Soc. (2006).
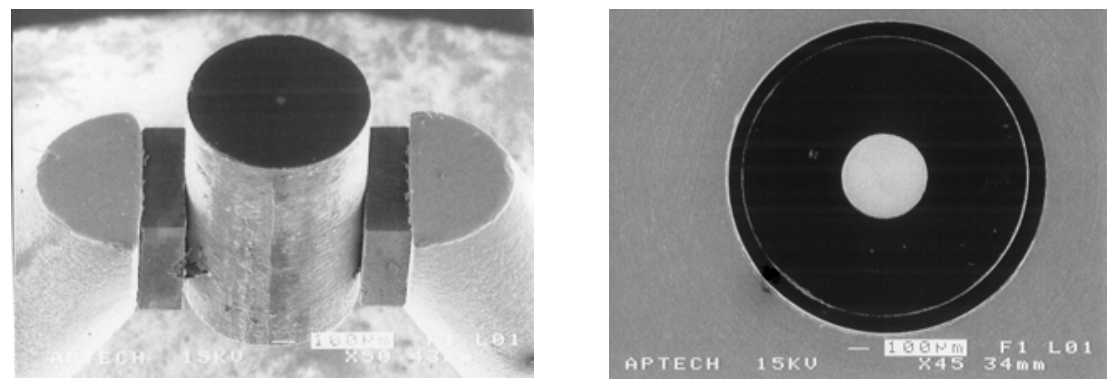

Fig. 1. (a) Vogel mount showing graphite guard ring and $100 \mu \mathrm{m} \mathrm{HfC(100)} \mathrm{cathode.} \mathrm{(b)} \mathrm{View} \mathrm{of} \mathrm{a} 0.25 \mathrm{~mm}$ radius $\mathrm{HfC}(100)$ cathode surrounded by graphite guard ring and centered in Pierce type housing.

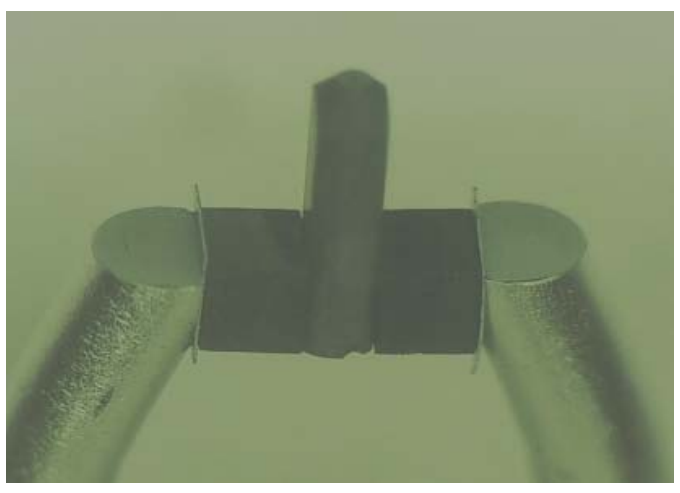

Fig. 2. Vogel mount showing central carbon embedded crystalline cathode, pyrolytic carbon heater blocks, the barrier or shim, and finally the Mo/Re clamping posts.

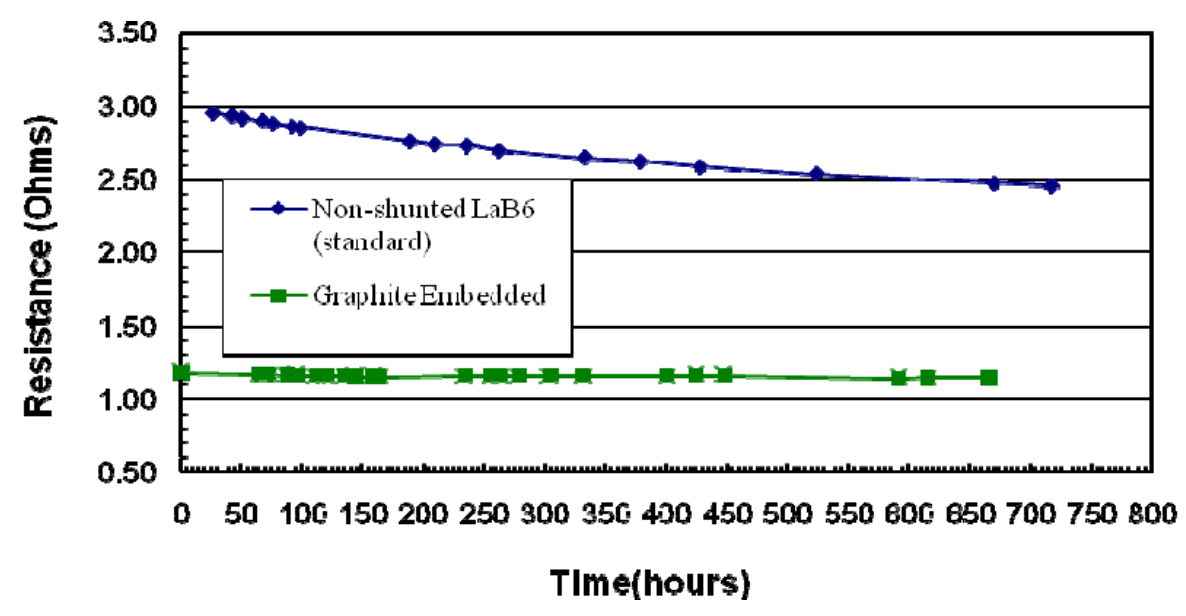

Fig. 3. Emission current over time showing longer term stability for a standard LaB6 cathode (top curve) and for the cathode material embedded in graphite (bottom curve). 